\title{
ON SOME VARIATIONAL INEQUALITIES FOR NONCLASSICAL TYPE OPERATORS
}

\author{
SERGEY GLAZATOV \\ Institute of Mathematics, Russian Academy of Sciences, Siberian Branch \\ Universitetskii Prosp. 4, 630090 Novosibirsk, Russia
}

The purpose of this paper is to make a brief review of results obtained in the theory of variational inequalities for nonclassical operators, namely, of degenerate hyperbolic and variable type.

1. Introduction. The theory of variational inequalities for elliptic and parabolic type operators is quite developed now and there exist many applications of this theory to various problems arising in mechanics, physics, electrodynamics etc. [4].

There are rather fewer papers considering variational inequalities for hyperbolic type and variable type operators, though there are, at least, two reasons for investigating such problems. The first one is to extend the theory of variational inequalities to a wider class of operators, and the second is the well-known phenomenon in the theory of initial-boundary value problems (IBVP) for nonlinear hyperbolic equations, namely, the blow-up of smooth solutions at finite time (the so-called "gradient catastrophe").

Let us present three examples. Consider three strictly hyperbolic equations in the cylinder $Q_{T}=(0, T) \times D$, where $D \subset \mathbb{R}^{n}$ is a bounded domain with smooth boundary $\partial D$ :

$$
\begin{aligned}
& L_{0} u \equiv u_{t t}-\Delta_{x} u+u^{2}=f(x, t), \\
& L_{1} u \equiv u_{t t}-\sum_{i=1}^{n} \frac{\partial}{\partial x_{i}}\left(a_{i}\left(u_{x_{i}}\right)\right)=f(x, t), \\
& L_{2} u \equiv u_{t t}-\Delta_{x} u+\varphi(u) u_{t}=f(x, t) .
\end{aligned}
$$

The following IBVPs can be posed: find a smooth solution of (1.1), (1.2) or 
(1.3) in $Q_{T}$, satisfying the initial-boundary value conditions

$$
\begin{aligned}
u(x, 0) & =u_{0}(x), \\
u_{t}(x, 0) & =u_{1}(x), \\
\left.u\right|_{\partial D \times(0, T)} & =0 .
\end{aligned}
$$

It is well-known that the IBVP (1.1), (1.4)-(1.6) has no global smooth solution for some smooth functions $u_{0}(x)$ and $u_{1}(x)$ [11]. It is necessary to use the method of "potential well" in order to obtain global smooth solutions of (1.1), (1.4)-(1.6), i.e. "smallness" (in appropriate norms) both of initial data and of the right-hand side $f(x, t)$ of (1.1) is required.

In the case of the IBVP (1.2), (1.4)-(1.6) there is an example [7] demonstrating that no "smallness" requirements can "improve" this problem: we can have a gradient catastrophe for arbitrarily small initial data.

In the case of the IBVP (1.3), (1.4)-(1.6) the existence or nonexistence of a global smooth solution depends on the sign of $\varphi(s)$, namely, if $\varphi(s) \geq \delta>0$, then there exists a global smooth solution for any smooth initial data and for any smooth right-hand side, but if $\varphi(s)$ may change its sign, then in general a blow-up may occur.

It was found out $[1,2,12]$ that in those three cases one can pose another problem - of finding a global smooth solution of a variational inequality with an appropriate restriction on $u_{t}$, instead of posing the IBVP. Smooth global solutions of the variational inequalities corresponding to equations (1.1)-(1.3) exist for any smooth initial data and for any smooth right-hand sides without any additional requirements, and these solutions satisfy (1.4)-(1.6).

Now we recall [11] how one can pose a problem of finding a solution of the variational inequality for the linear wave operator, for example. In the domain $Q_{T}$ we consider the wave equation

$$
\square u \equiv u_{t t}-\Delta_{x} u=f(x, t)
$$

and the boundary conditions (1.4)-(1.6). Here $f(x, t)$ is a given function.

Let us introduce a convex set of restrictions $K \subset \stackrel{\circ}{W}_{2}^{1}(D)$ closed in $\stackrel{\circ}{W}_{2}^{1}(D)$. The problem consists in finding a function $u(x, t) \in L^{\infty}\left(0, T ; \stackrel{\circ}{W}_{2}^{1}\right)$ such that

(a) $u_{t} \in L^{\infty}\left(0, T ; \stackrel{\circ}{W}_{2}^{1}\right), u_{t t} \in L^{\infty}\left(0, T ; L^{2}\right)$

(b) $u(x, t)$ satisfies (1.4)-(1.6),

(c) $u_{t}(\cdot, t) \in K$ a.e. on $[0, T]$,

(d) for all $t \in[0, T]$ and for any $v \in L^{\infty}\left(0, T ; \stackrel{\circ}{W}_{2}^{1}\right)$ such that $v(\cdot, t) \in K$ a.e. on $[0, T]$ we have the variational inequality

$$
\int_{0}^{t}\left(\square u-f, v-u_{\tau}\right)_{0} d \tau \geq 0 .
$$

From now on, $(\cdot, \cdot)_{0}$ denotes either the inner product in $L^{2}(0,1)$ or the duality between some function spaces. The above problem has a unique solution [11]. 
We should note that the choice of the convex set $K$ of restrictions and of appropriate function spaces essentially depends on the type of the hyperbolic operator, especially in the nonlinear case.

In the 80's a series of papers $[1-3,8-10,12]$ appeared in which hyperbolic variational inequalities were treated. In these papers various particular cases of the general operator

$$
L u \equiv k(u) u_{t t}-\sum_{i=1}^{n} \frac{\partial}{\partial x_{i}}\left(a_{i}\left(u_{x_{i}}\right)\right)+f\left(x, t ; u, u_{t}\right)
$$

were considered together with the initial-boundary value conditions (1.4)-(1.6). Sufficient smoothness of the coefficients of (1.8) was required. Existence theorems for smooth global solutions of the corresponding variational inequalities were proved by a correct choice of function spaces and sets of restrictions on $u_{t}$ in each case. In all those papers the following conditions on the coefficients of (1.8) were assumed: $k(s) \geq \delta>0, a_{i}^{\prime}(s) \geq \delta>0, i=1, \ldots, n$ (which yields strict hyperbolicity of $L)$ and $k(s) \equiv k_{0}>0$ if $n>1$.

2. Variational inequality for some degenerate hyperbolic operators. In this section we deal with operators which fail to be strictly hyperbolic. Consider the rectangular domain $Q=(0, T) \times(0,1)$, the operator

$$
M_{0} u \equiv k\left(u_{t}\right) u_{t t}-u_{x x}-f(x, t)
$$

in this domain and the initial-boundary value conditions

$$
\begin{aligned}
\left.u\right|_{t=0} & =u_{0}(x), \\
\left.u_{t}\right|_{t=0} & =u_{1}(x), \\
\left.u\right|_{x=0} & =\left.u\right|_{x=1}=0 .
\end{aligned}
$$

Here $k(s) \geq 0$ and $k(s)$ can be zero only at isolated points; moreover, $k(s)$ satisfies the Lipschitz condition on every compact subset $K \subset \mathbb{R}$ (with a Lipschitz constant depending on $K$ ).

Let us introduce two primitives:

$$
A(s)=\int_{0}^{s} k(\sigma) \sigma d \sigma, \quad B(s)=\int_{0}^{s} \sqrt{k(\sigma)} d \sigma .
$$

We assume that $A(s)$ is a convex function, and $A(s) \geq C_{0}|s|^{p}(p>1)$ for any $s \in \mathbb{R}\left(C_{0}\right.$ is a positive constant).

Let us introduce the convex set of restrictions

$$
K=\left\{z \in \stackrel{\circ}{W}_{2}^{1}(0,1):\left|z_{x}\right| \leq 1 \text { a.e. on }[0,1]\right\} .
$$

The following theorem is valid:

TheOrem 1 [5]. Assume that all the above conditions on $k(s)$ hold and that $f, f_{t} \in L^{p^{\prime}}(Q)$ (here $p^{\prime} \in(1, \infty)$ is such that $\left.1 / p+1 / p^{\prime}=1\right), u_{0} \in \stackrel{\circ}{W}_{4}^{1}(0,1)$, 
$u_{1} \in K$. Then there exists a function $u(x, t) \in L^{\infty}\left(0, T ; \stackrel{\circ}{W}_{4}^{1}\right)$ such that $u_{t} \in$ $L^{\infty}\left(0, T ; \stackrel{\circ}{W}_{4}^{1}\right),(\partial / \partial t)\left(B\left(u_{t}\right)\right) \in L^{2}(Q), u(x, t)$ satisfies $(2.2)-(2.4), u_{t}(, t) \in K$ a.e. on $[0, T]$ and for any $v(x, t) \in L^{\infty}\left(0, T ; \stackrel{\circ}{4}_{4}^{1}\right)$ such that $v(\cdot, t) \in K$ a.e. on $[0, T]$, the variational inequality

$$
\int_{0}^{t}\left(M_{0} u, v-u_{\tau}\right)_{0} d \tau \geq 0
$$

is valid for all $t \in[0, T]$.

The following question arises: what is meant by saying that $u(x, t)$ satisfies the initial condition (2.3)? Unfortunately, we can only show that $B\left(u_{t}(t)\right) \rightarrow B\left(u_{1}\right)$ in $L^{2}(0,1)$ as $t \rightarrow 0+$, so the second initial condition is fulfilled not in the usual sense. But in the case when $k(s) \geq \delta>0$ (i.e. $M_{0}$ is strictly hyperbolic) one can easily derive that $u_{t}(t) \rightarrow u_{1}$ in $L^{2}(0,1)$ as $t \rightarrow 0+$.

The proof of Theorem 1 was published in [5]. The penalty method, " $\varepsilon$-regularization", Galerkin methods and a special technique of obtaining a priori estimates were used.

3. Variational inequality for a variable type operator. The nonlinear equation of variable type

$$
M_{1} u \equiv u_{x} u_{x x}-u_{y y}=f(x, y)
$$

arises in transsonic gas dynamics. It is usually called the Karman equation.

It is very difficult to pose a correct boundary value problem for (3.1) because of its nonlinearity, variable type and of the absence of any a priori estimates even for smooth solutions. There is a paper [13] by S. Pyatkov in which a boundary value problem of Neumann type was posed for (3.1). He obtained quite a nice solution of this problem for which $u_{x} \leq 0$. We shall treat a case "opposite", in some sense, namely, (3.1) in the rectangular domain $Q=(0, L) \times(0,1)$ together with the initial-boundary value conditions

$$
\begin{aligned}
u(0, y) & =\varphi(y), \\
u_{x}(0, y) & =\psi(y), \\
u(x, 0) & =u(x, 1)=0 .
\end{aligned}
$$

Note that we pose "hyperbolic" initial-boundary value conditions despite the fact that (3.1) is actually a stationary equation. Here $x$ plays the role of the "time" variable and $y$ is the "space" variable.

Let us introduce two sets of restrictions:

$$
\begin{aligned}
& K_{1}=\left\{z \in \stackrel{\circ}{W}_{4}^{1}(0,1): z \geq 0 \text { a.e. on }[0,1]\right\}, \\
& K_{2}=\left\{z \in \stackrel{\circ}{W}_{4}^{1}(0,1):\left|z_{y}\right| \leq 1 \text { a.e. on }[0,1]\right\} .
\end{aligned}
$$

The purpose of the first set $K_{1}$ is evident: we want to fix the type of (3.1), namely, it is degenerate hyperbolic on the class of solutions for which $u_{x} \geq 0$ 
a.e. on $Q$. There is an example [1] where the variational inequality for a second order hyperbolic operator nonlinear in the principle part with restrictions only on the first derivative $u_{t}$ occurs to be unsolvable. This is the reason why we must introduce the second restriction (namely $\left|u_{x y}\right| \leq 1$ ) on the mixed second order partial derivative.

The following theorem is valid.

Theorem 2 [6]. Assume that $f, f_{x} \in L^{3 / 2}(Q), \varphi \in \stackrel{\circ}{W}_{4}^{1}(0,1), \psi \in K_{1} \cap K_{2}$. Then there exists $u(x, y) \in L^{\infty}\left(0, L ; \stackrel{\circ}{W}_{4}^{1}\right)$ such that $u_{x} \in L^{\infty}\left(0, L ; \stackrel{\circ}{W}_{4}^{1}\right)$ and $(\partial / \partial x)\left(u_{x}^{3 / 2}\right) \in L^{2}(Q), u(x, y)$ satisfies $(3.2)-(3.4), u_{x}(x, \cdot) \in K_{1} \cap K_{2}$ a.e. on $[0, L]$ and for all $x \in[0, L]$ and for any $v(x, y) \in L^{\infty}\left(0, L ; \stackrel{\circ}{W}_{4}^{1}\right)$ such that $v(x, \cdot) \in$ $K_{1} \cap K_{2}$ a.e. on $[0, L]$ the variational inequality

$$
\int_{0}^{x}\left(M_{1} u-f, v-u_{\xi}\right)_{0} d \xi \geq 0
$$

is valid.

The proof of Theorem 2 was published in [6]. It is analogous to the proof of Theorem 1, except that it is possible to derive that $u_{x}(x) \rightarrow \psi$ in $L^{2}(0,1)$ as $x \rightarrow 0+$, i.e. the initial conditions are satisfied in the usual sense.

To conclude, we remark that it would be interesting to consider multidimensional problems like (2.1) and (3.1), but our special technique of obtaining a priori estimates and using embedding theorems works in the one-dimensional case only.

\section{References}

[1] A. B. Aliev, Variational inequalities for quasilinear hyperbolic type operators, Mat. Zametki 42 (3) (1987), 369-380 (in Russian).

[2] - One sided problems for quasilinear hyperbolic operators in function spaces, Dokl. Akad. Nauk SSSR 297 (2) (1987), 271-275 (in Russian).

[3] -, Global solvability of one-sided problems for quasilinear hyperbolic type equations, ibid. 298 (5) (1988), 1033-1036 (in Russian).

[4] G. Duvaut et J.-L. Lions, Les inéquations en mécanique et en physique, Dunod, Paris 1972 .

[5] S. Glazatov, On a class of quasilinear hyperbolic inequalities, in: Some Applications of Functional Analysis to Problems of Mathematical Physics, Inst. Math., Siberian Branch Acad. Sci. U.S.S.R., Novosibirsk 1990, 67-80 (in Russian).

[6] -, A variational inequality connected with a variable type nonlinear equation, in: Dynamics of Continuous Media 99, Inst. Hydrodynamics, Siberian Branch Acad. Sci. U.S.S.R., Novosibirsk 1990, 18-33 (in Russian).

[7] S. Klainerman and A. Majda, Formation of singularities for wave equations, Comm. Pure Appl. Math. 33 (1980), 241-264.

[8] R. Landes, Quasilinear hyperbolic variational inequalities, Arch. Rational Mech. Anal. 91 (3) (1986), 267-272.

[9] N. A. Lar'kin, On global solutions of nonlinear hyperbolic inequalities, Dokl. Akad. Nauk SSSR 250 (4) (1980), 806-809 (in Russian). 
[10] N. A. Lar'kin, A one-sided problem for a nonlocal quasilinear hyperbolic equation arising in the theory of elasticity, ibid. 274 (6) (1984), 1341-1344 (in Russian).

[11] J.-L. Lions, Quelques méthodes de résolution des problèmes aux limites non linéaires, Dunod, Paris 1969.

[12] F. G. Maksudov, A. B. Aliev and D. M. Takhirov, A one-sided problem for a quasilinear equation of hyperbolic type, Dokl. Akad. Nauk SSSR 258 (4) (1981), 789-791 (in Russian).

[13] S. Pyatkov, Solvability of boundary value problems for a nonlinear degenerate elliptic equation, in: Applications of Functional Analysis to Nonclassical Equations of Mathematical Physics, Inst. Math., Siberian Branch Acad. Sci. U.S.S.R., Novosibirsk 1988, 102-117 (in Russian). 